\title{
Safety and Efficacy of Early Endotherapy in Management of Pancreatic Ascites: Western Indian Experience
}

\author{
Mayur G. Gattani ${ }^{11}$ Shamshersingh G. Chauhan ${ }^{1}$ Pratik R. Sethiya ${ }^{1}$ Pooja C. Chandak ${ }^{2}$ \\ Saiprasad G. Lad ${ }^{1}$ Gaurav K. Singh ${ }^{1} \quad$ Kailash M. Kolhe ${ }^{1}$ Harshad B. Khairnar $^{1}$ Vikas R. Pandey $^{1}{ }^{10}$ \\ Meghraj A. Ingle ${ }^{1}$

\footnotetext{
${ }^{1}$ Department of Gastroenterology, Lokmanya Tilak Municipal Medical College \& General Hospital, Mumbai, India

2 Department of Radiology, Lokmanya Tilak Municipal Medical College

\& General Hospital, Mumbai, India
} \\ Address for correspondence Vikas R. Pandey, MD DM, Department of \\ Gastroenterology, Lokmanya Tilak Municipal Medical College \& \\ General hospital, Sion, Mumbai 400022, India \\ (e-mail: drvikas_pandey@rediffmail.com).
}

J Digest Endosc 2021;12:183-189.
Abstract
Keywords
- cholangiopan- creatography
- endoscopic retrograde
- Magnetic resonance
- ascites
- pancreatitis
- acute necrotizing
- sphincterotomy

Background Pancreatic ascites is rare but a known complication of pancreatitis. We aimed to study the timings, safety, and efficacy of therapeutic approaches in its management and the outcomes.

Methods We retrospectively studied patients with pancreatic ascites managed in the past 5 years at a single tertiary care center. Therapeutic approaches included conservative therapy, early endoscopic therapy, and surgery. We used descriptive statistics to summarize characteristics of the study population, and performed univariate and binary logistic regression analyses to compare treatment outcomes.

Results Of the 125 patients screened, 70 (male, 81.4\%) were included. Disruption in the pancreatic duct (PD) was seen in $51.4 \%$ of patients on magnetic resonance cholangiopancreatography (MRCP) and $73.3 \%$ of patients on endoscopic retrograde cholangiopancreatography (ERCP). The PD in the body region (46.7\%) was the most frequent site of disruption. Early endotherapy included a stent bridging the disruption site in $63.3 \%$ of patients and sphincterotomy in $76.7 \%$ of patients with a median time to ERCP from symptom onset being 8.5 days. The success rate in early endotherapy was $81.7 \%$, while the recurrence rate was $8 \%$. For conservative therapy only, the success rate was $60 \%$ with recurrence in two-thirds. The variables crucial in the success of endotherapy were a partial disruption $(p<0.001)$, ductal disruption site $(p=0.004)$, sphincterotomy $(p=0.013)$, and a bridging stent $(p=0.001)$. Significant pancreatic necrosis $(p<0.001)$ and intraductal calculi $(p=0.002)$ were the factors responsible for failure in endotherapy.

Conclusions Early endotherapy is safe and effective in the treatment of pancreatic ascites. The efficacy of endotherapy is augmented by PD stenting combined with pancreatic sphincterotomy and a bridging stent.
DOI https://doi.org/ 10.1055/s-0041-1741515. ISSN 0976-5042. (c) 2022. Society of Gastrointestinal Endoscopy of India. All rights reserved.

This is an open access article published by Thieme under the terms of the Creative Commons Attribution-NonDerivative-NonCommercial-License, permitting copying and reproduction so long as the original work is given appropriate credit. Contents may not be used for commercial purposes, or adapted, remixed, transformed or built upon. (https://creativecommons.org/ licenses/by-nc-nd/4.0/)

Thieme Medical and Scientific Publishers Pvt. Ltd., A-12, 2nd Floor, Sector 2, Noida-201301 UP, India 


\section{Introduction}

Pathophysiology of pancreatic ascites involves the leak of pancreatic secretions in the peritoneal cavity. ${ }^{1,2}$ In patients with chronic pancreatitis (CP), the pseudocysts generally have a less sturdy fibrinous wall. This leads to the leakage of secretions from the duct disruption in the peritoneal cavity through the pseudocyst. ${ }^{2,3}$ In cases of acute necrotizing pancreatitis (ANP), parenchymal necrosis can result in duct disruption (anterior) and resultant ascites. ${ }^{2,3}$ Severity in these cases mainly depends on the site and degree of disruption. ${ }^{3-6}$ Spontaneous resolution is seen in most of the mild varieties, while increased rates of morbidity and mortality are seen in persistent cases and those with infection. ${ }^{6-8}$ Diversion of pancreatic secretions from the disrupted site to the bowel commonly by pancreatic endotherapy and less commonly by surgery helps in healing the disruption. ${ }^{1,2}$ However, patients may be subjected to conservative therapy only, which may be effective in a few cases though. ${ }^{9}$ As this condition is rare, studies comparing various therapeutic approaches are unavailable. Pooled analysis of various studies has shown a success rate of 60 to $92 \%$ for endotherapy, 67 to $89 \%$ for surgery, while the success rate of conservative treatment is mostly unknown. ${ }^{10}$

Prognosis depends on the etiology and certainty of the indications for treatment. ${ }^{6-10}$ Endotherapy has improved the clinical outcomes in most patients, but with variable success rates. The latest data show that endotherapy is associated with lesser mortality, decreased length of hospital stay, and lower recurrence rates compared with conservative and surgical approaches. ${ }^{7-11}$

As ascites is a rare complication of pancreatitis, there is very limited published literature on its clinical aspects and timings of therapeutic approaches. Thus, we studied the clinical profile, efficacy of different management strategies and outcomes in patients with pancreatic ascites.

\section{Methods}

This single-center observational study was performed after approval from the Institutional Ethics Committee (IEC) 34/20). Medical records of all patients with pancreatitis and ascites reviewed at a tertiary care hospital from 2016 to 2020 were accessed for data retrieval including demography, history, clinical details, laboratory investigations, imaging, treatment, and follow-up. The inclusion criteria were symptomatic ascites with or without pleural effusion, amylase level in ascitic fluid more than serum amylase level, either chronic pancreatitis with exacerbation or acute pancreatitis, and no other etiology for fluid collection. Exclusion criteria were mixed etiology of ascites, minimal ascites or effusion (non-tappable), treatment required for associated conditions only (e.g., drainage of peri-pancreatic fluid collections only, intractable pancreatic pain requiring stenting), non-availability of complete records, and lack/technical failure of endoscopic retrograde cholangiopancreatography (ERCP) or magnetic resonance cholangiopancreatography
(MRCP). The flow diagram of the study including our management protocol is shown in - Fig. 1.

\section{Nomenclature and Definitions}

Pancreatic ascites was defined as free-fluid in the peritoneal cavity seen on ultrasonography (USG) or computed tomography (CT) scan of the abdomen with an amylase level more than the serum amylase level in a patient with benign pancreatic disorder. ${ }^{7,12}$ Pancreatic fluid collection (PFC) was the term used for any pancreatic/peri-pancreatic collection developing secondary to either fluid leakage from the pancreas or liquefaction of pancreatic necrosis with discrimination as WON and pseudocyst as seen on contrast-enhanced CT (CECT) scan as per the revised Atlanta classification. ${ }^{13}$ Pancreatic duct (PD) disruption was defined by extravasation of contrast outside the pancreatic ductal system on fluoroscopy during ERCP or any discontinuity of PD noticed on MRCP. ${ }^{7,14-16}$ It was defined as partial when the PD proximal to the site of disruption was opacified on ERCP and complete when the PD proximal to disruption was not opacified. ${ }^{8}$ Pancreatitis was classified as mild, moderately severe, or severe according to the revised Atlanta classifica$\operatorname{tion}^{13}$ and the extent of necrosis in the parenchyma was categorized as $<30 \%$ or $>30 \%$, based on the modified CT severity index (CTSI). ${ }^{17}$ Due to paucity and heterogeneity of data on the subject, we defined rapidly refilling ascites as recurrence of ascites within 48 hours of a therapeutic ascitic tap with an intent to mobilize all ascitic fluid, with amount enough to cause discomfort to the patient or moderate levels as seen on ultrasound. We defined early endotherapy as PD stenting done within 2 weeks of symptom onset for pancreatic ascites as sequelae to ANP or CP. We defined successful therapy as a resolution of ascites on imaging (mostly USG) and a repeat pancreatogram at 6 weeks not showing any disruption. We defined failure of therapy as persistence or partial resolution of ascites at 6 weeks. Recurrence was defined as ascites with another PFC in the same site or a new PFC elsewhere as seen on USG or cross-sectional imaging study, along with associated symptoms after successful resolution. ${ }^{18}$ Procedure-related complications were defined as recommended by Cotton et al. ${ }^{19}$

\section{Management Protocol}

All included patients had undergone blood tests including complete blood count, liver and renal function tests, serum amylase and lipase levels, serum triglycerides, serum calcium levels, ascitic fluid analysis including amylase levels, CECT of the abdomen for PFCs, the extent of pancreatic necrosis, and pancreatic duct dilatation followed by MRCP to delineate ductal anatomy. The CT scan was done 72 hours after the onset of symptoms of pancreatitis and MRCP was done when the diagnosis of pancreatic ascites was confirmed on ascitic fluid analysis. All patients were initially started on conservative therapy in the form of nasojejunal ( $\mathrm{NJ}$ ) tube feeding, octreotide $100 \mu \mathrm{g}$ tid intravenously, and intravenous fluids as necessary with therapeutic paracentesis. MRCP was done in the patients only after therapeutic paracentesis. Those who did not have both rapidly refilling ascites and did not demonstrate a 


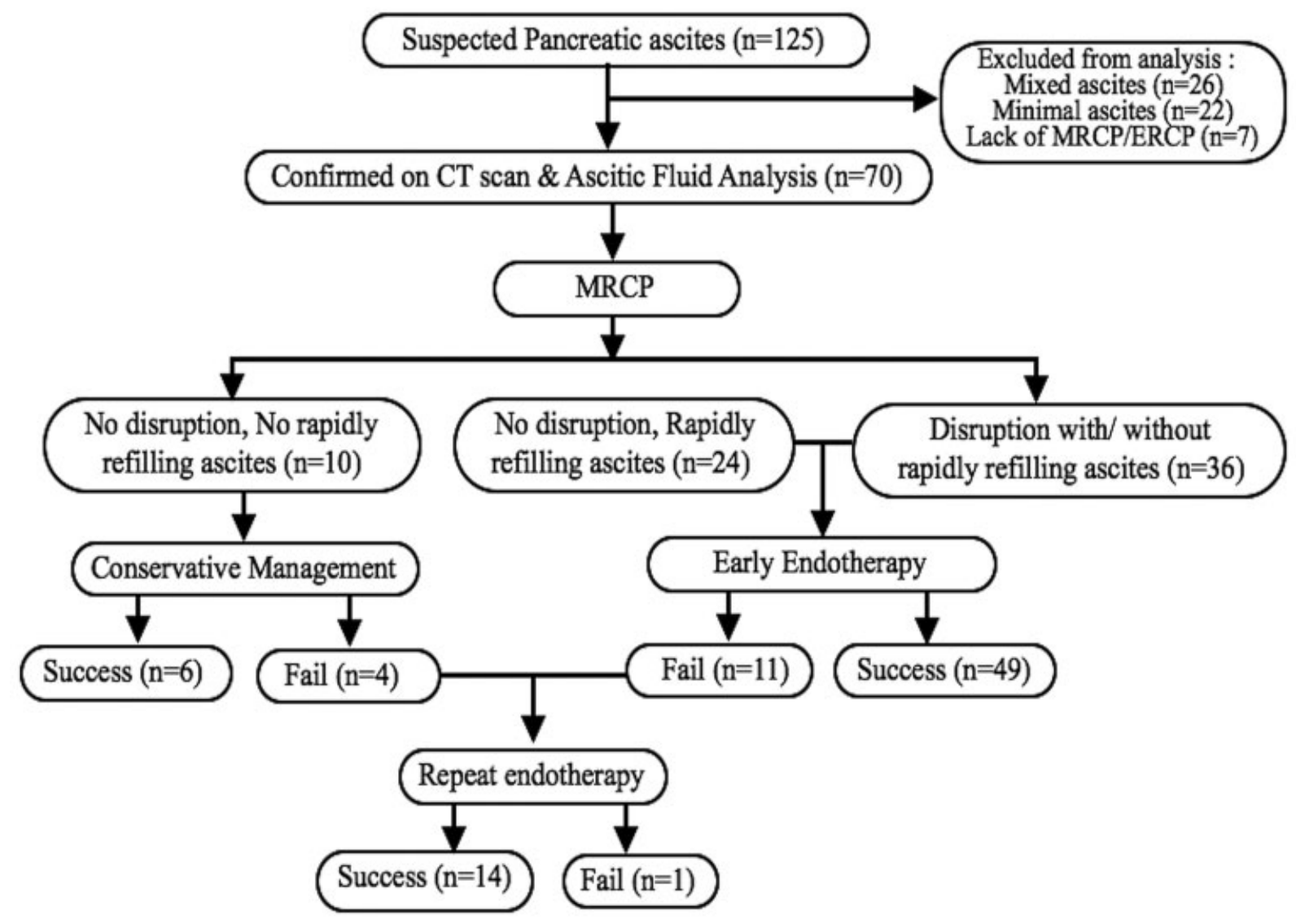

Fig. 1 The flow diagram and management protocol of the study.

disruption on MRCP were continued on conservative therapy for 6 weeks. Those who had rapidly refilling ascites causing respiratory discomfort with/without a demonstrable disruption on MRCP were taken up for endotherapy immediately and intravenous octreotide was stopped post-procedure. NJ tube feeding and intravenous fluid therapy were continued as per patient requirements. Written informed consent was taken from patients before any intervention.

During endotherapy, patients were sedated with intravenous propofol and hyoscine butyl bromide for duodenal relaxation. ERCP was performed in patients using the standard technique using a TJF 150 (Olympus Optical, Tokyo, Japan) side-viewing duodenoscope by a single expert endoscopist with more than 7 years of experience. The site of disruption was located on pancreatogram along with any concomitant strictures or stones. Pancreatic sphincterotomy was done in some patients as per the discretion of the endoscopist and a straight plastic pancreatic stent was put up to or across the disruption site. When a disruption was not found, a longer stent was used to reach up to the tail region. At 6 weeks, a repeat pancreatogram was obtained to document resolution. Stent removal was done at the same time if successful resolution or exchanged if the disruption was persistent. Patients were referred for surgery only if ERCP failed or was not technically feasible or if any serious complication developed.

\section{Outcome Data}

Clinical evaluation of patients was done followed by imaging (USG or CT) until complete resolution of ascites or any alternative treatment approach was needed. Follow-up data of all patients were searched for any recurrence, days of hospital stay, days for resolution of ascites, and mortality. Retrieval of data related to complications post endotherapy was also done.

\section{Statistical Analysis}

Continuous data are presented as median with a range from minimum to maximum values or as mean \pm standard deviation (SD). Categorization of the patients was done on basis of the successful or failed outcome of treatment. Chi-square/Fischer's exact test was used to determine the univariate relationship of every independent variable to the outcome under study. Binary logistic regression analysis was used to study the simultaneous effects of the variables on the likelihood that each outcome would occur. $p<0.05$ was considered statistically significant. Data analysis was performed using the statistical software SPSS 20.0 (SPSS Inc, Chicago, IL).

\section{Results}

Of the 125 patients screened over a period of 5 years, 70 patients (male $=57 ; 81.4 \%$ ) met the inclusion criteria. The 
Table 1 Baseline characteristics of patients

\begin{tabular}{|l|l|}
\hline Characteristics & $\mathbf{n = 7 0}(\%)$ \\
\hline Male:female & $4.4: 1$ \\
\hline Age in years (mean) & $41.38( \pm 10.67)$ \\
\hline Type of pancreatitis: & \\
\hline Acute & $26(37.1 \%)$ \\
\hline Chronic & $44(62.9 \%)$ \\
\hline Etiology of pancreatitis: & \\
\hline Alcoholic & $55(78.6 \%)$ \\
\hline Idiopathic & $9(12.8 \%)$ \\
\hline Gall stones & $3(4.3 \%)$ \\
\hline Traumatic & $3(4.3 \%)$ \\
\hline Patients with diabetes & $12(17.1 \%)$ \\
\hline Ascites alone & $60(85.7 \%)$ \\
\hline Ascites with pleural effusion & $10(14.3 \%)$ \\
\hline Mean ascitic fluid amylase (IU/mL) & $6543.81( \pm 2361.51)$ \\
\hline Mortality & $1(1.43 \%)$ \\
\hline Mean hospital stay (days) & $44.86( \pm 8.38)$ \\
\hline
\end{tabular}

baseline characteristics of the study population are shown in - Table 1. A total of 26/70 (37.1\%) patients had ANP and $44 / 70(62.9 \%)$ had CP. The underlying etiology of pancreatitis was alcohol in 55/70 (78.6\%) patients. The most common presenting symptom was abdominal distension with mild abdominal discomfort, followed by abdominal pain and weight loss. Twelve patients (17.1\%) were detected to be diabetic. Endotherapy was done in $60 / 70$ patients (85.7\%), out of which 20 had ANP and 40 had CP. The remaining 10/70 patients (14.3\%) were conservatively managed, out of which 4 had ANP and 6 had CP.

On a CECT scan, one or more PFCs were documented in $62 / 70$ (88.6\%) patients (- Fig. 2). Of the 26 patients with ANP, the severity of pancreatitis (as per modified CT severity index) was moderately severe in 16 patients $(61.5 \%)$ and severe in 10 patients (38.5\%), while the extent of necrosis was $<30 \%$ in 22 and $>30 \%$ in 4 patients. On MRCP, intraductal calculi were present in $16 / 44$ patients (36.4\%) of CP. MRCP did not demonstrate a disruption from the PD in 34/70 patients (48.6\%) of pancreatic ascites. Ten of these patients were managed conservatively only as they did not have rapidly re-filling ascites. The remaining 24 patients underwent ERCP and disruption was appreciated additionally in 8 of these patients. Disruption in PD was seen in 36/70 (51.4\%) patients on MRCP. All these patients underwent ERCP and disruptions were appreciated on pancreatogram in all. Thus in total, disruption was demonstrated in $44 / 60$ patients $(73.3 \%$ ) on ERCP. Complete disruption was seen in 2 patients while the rest of the 42 patients had a partial disruption. The PD in the body region (28/60 patients, $46.7 \%$ ) was the most frequent site of disruption ( - Fig. 2), then head (10/60 patients, 16.7\%) and tail (6/60 patients, $10 \%)$. The disruption site was obscured in $16 / 60$ patients (26.7\%) on ERCP. The median
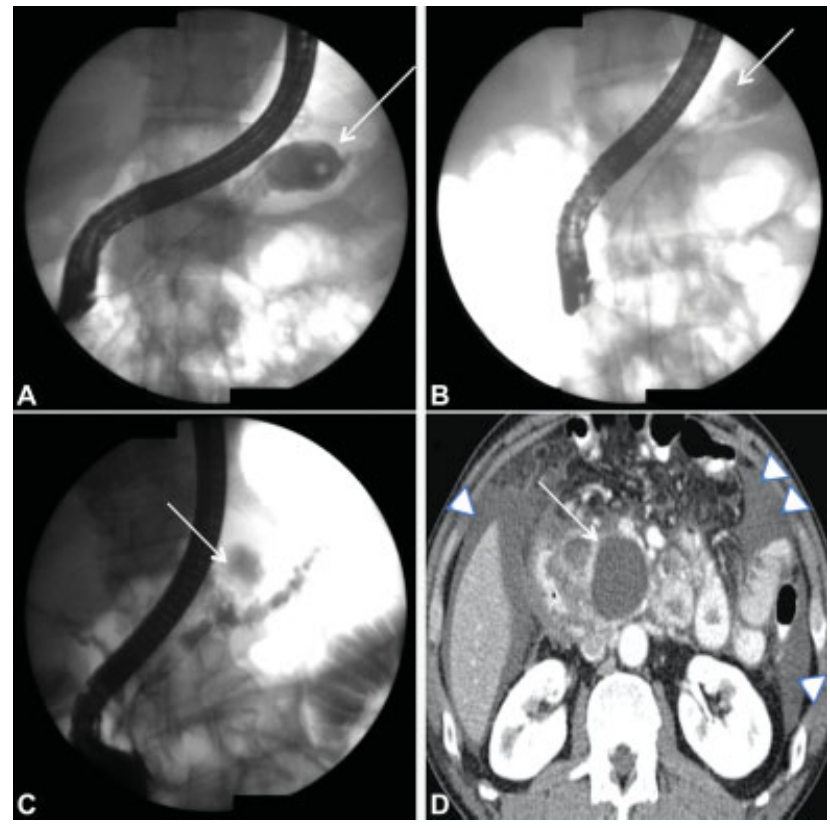

Fig. 2 CT Scan and ERCP pancreatograms of patients: (A) A pancreatogram showing disruption in the tail region (arrow). (B) Fluoroscopic image showing stent bridging disruption site and in the cavity (arrow). (C). A pancreatogram showing disruption in the body region (arrow). (D) Corresponding CT scan showing a collection in the body (arrow) with ascites (arrowheads).

time to ERCP from symptom onset was 8.5 days (range: $6-12$ days).

Characteristics of endotherapy are shown in - Table 2. During endotherapy, a stent was placed beyond the disruption (i.e., proximal to the disruption site) in 39/44 (88.6\%) patients. In 5/44 (11.4\%) patients, the stent was not passed beyond the disruption, i.e., placed distal to the PD disruption site across the papilla. Empirical stent placement up to the tail region was done in 16 patients in whom the location of PD disruption was obscured. Sphincterotomy was done in $46 / 60$ (76.7\%) patients. Patients were assessed at 6 weeks for resolution of ascites. Endotherapy was successful in 49/60 (81.7\%) patients. Bridging the disruption with stent during ERCP $(p=0.001)$ and concomitant pancreatic sphincterotomy ( $p=0.013$ ) were associated with success in endotherapy as depicted in -Table 3. Similarly, the site of PD disruption was an important factor associated with the outcome of endotherapy. Disruptions present in the body and head

Table 2 Characteristics of the endotherapy

\begin{tabular}{|l|l|}
\hline Endotherapy characteristics & $\mathbf{n = 6 0}$ \\
\hline $\begin{array}{l}\text { Number of attempts for stent } \\
\text { placement (median) }\end{array}$ & $1(1-3)$ \\
\hline Median stent diameter (Fr) & $7(5-10)$ \\
\hline Median stent length (cm) & $7(5-12)$ \\
\hline $\begin{array}{l}\text { Stent relation to disruption site, } n(\%) \\
\text { - Bridging }\end{array}$ & $38(63.3 \%)$ \\
\hline Transpapillary only & $22(36.7 \%)$ \\
\hline Sphincterotomy & $46(76.7 \%)$ \\
\hline
\end{tabular}


Table 3 Significance of factors affecting endotherapy outcome, $n=60$

\begin{tabular}{|c|c|c|c|c|}
\hline & Parameters & $\begin{array}{l}\text { Successful } \\
\text { endotherapy } \\
(n=49)\end{array}$ & $\begin{array}{l}\text { Failed } \\
\text { endotherapy } \\
(n=11)\end{array}$ & $p$-value \\
\hline \multirow[t]{2}{*}{ Clinical profile } & Ascites & 43 & 8 & \multirow[t]{2}{*}{0.344} \\
\hline & Ascites with pleural effusion & 6 & 3 & \\
\hline \multirow[t]{2}{*}{ Type of pancreatitis } & ANP & 15 & 5 & \multirow[t]{2}{*}{0.481} \\
\hline & $\mathrm{CP}$ & 34 & 6 & \\
\hline \multirow[t]{4}{*}{ Underlying etiology } & Alcohol & 38 & 7 & \multirow[t]{4}{*}{0.118} \\
\hline & Idiopathic & 8 & 1 & \\
\hline & Gall Stones & 2 & 1 & \\
\hline & Trauma & 1 & 2 & \\
\hline \multirow[t]{2}{*}{ Extent of necrosis in ANPa } & $<30 \%$ & 15 & 0 & \multirow[t]{2}{*}{$<0.001$} \\
\hline & $>30 \%$ & 0 & 5 & \\
\hline \multirow[t]{2}{*}{ Intraductal calculi in $\mathrm{CP}^{\mathrm{b}}$} & Present & 10 & 6 & \multirow[t]{2}{*}{0.002} \\
\hline & Absent & 24 & 0 & \\
\hline \multirow[t]{4}{*}{ Disruption site } & Head & 10 & 0 & \multirow[t]{4}{*}{0.004} \\
\hline & Body & 26 & 2 & \\
\hline & Tail & 4 & 2 & \\
\hline & Obscured & 9 & 7 & \\
\hline \multirow[t]{3}{*}{ Type of disruption } & Complete & 0 & 2 & \multirow[t]{3}{*}{$<0.001$} \\
\hline & Partial & 40 & 2 & \\
\hline & Not seen & 9 & 7 & \\
\hline \multirow[t]{2}{*}{ Sphincterotomy } & Done & 41 & 5 & \multirow[t]{2}{*}{0.013} \\
\hline & Not done & 8 & 6 & \\
\hline \multirow[t]{3}{*}{ Stent position } & Disruption bridged & 36 & 2 & \multirow[t]{3}{*}{0.001} \\
\hline & Disruption not bridged & 4 & 2 & \\
\hline & Empirical & 9 & 7 & \\
\hline
\end{tabular}

${ }^{a}$ ANP, acute necrotizing pancreatitis.

${ }^{\mathrm{b}} \mathrm{CP}$, chronic pancreatitis.

$p<0.05$, significant.

region had more success in endotherapy than those present in the tail region $(p=0.004)$. This was likely because the former disruptions were comparatively easier to bridge by stenting than the latter. Similarly, endotherapy for partial disruptions was more successful than for complete or obscured disruptions ( $p \leq 0.001$ ). One patient had developed symptomatic, large WON abutting the stomach wall after trans-papillary stenting for ascites. It was drained transmurally in a subsequent session and also had required further two sessions of direct endoscopic necrosectomy (DEN).

Early endotherapy failed in $11 / 60(18.3 \%)$ patients ( 5 with ANP and 6 with $\mathrm{CP})$ at 6 weeks. Intraductal calculi $(p=0.049)$ in patients with CP and significant necrosis $>30 \%$ in ANP $(p=0.022)$ were the factors associated with failed endotherapy. A repeat ERCP with pancreatic duct stenting with a longer stent was done in 10 of these patients who responded while 1 patient who had undergone DEN died before being referred for surgery. No other direct complication of endoscopic therapy was seen.
The mean hospital stay was $44.86( \pm 8.38)$ days with a median follow-up period of 22 months (4-58 months). The success rate in early endotherapy was $81.7 \%$, out of which $8 \%$ had a recurrence and only $60 \%$ for conservative therapy, out of which two-thirds had a recurrence on follow-up. These patients then required treatment with pancreatic endotherapy.

\section{Discussion}

Our results showed that pancreatic ascites were present commonly in patients with more severe pancreatitis and associated PFCs. We demonstrated that conservative therapy has a very low success rate with high recurrence for pancreatic ascites and also that early endotherapy with stent placement is very effective in patients with a rapidly refilling pancreatic ascites associated with both acute and chronic pancreatitis. We showed that the site of disruption in the body region, stent bridging the disruption site, a concomitant sphincterotomy are the factors crucial for 
successful endotherapy and significant parenchymal necrosis, and the presence of pancreatic ductal calculi can negatively affect the outcome of endotherapy.

As pancreatic ascites are uncommon, the scientific evidence available currently, regarding its clinical profile, management, and outcomes is very limited. To our knowledge, there are no randomized controlled trials (RCTs) comparing the conservative, endoscopic, radiologic, or surgical treatment approaches as they are difficult to perform. The management of pancreatic ascites involves patient stabilization, delineating the PD, and locating the PD disruption site with its definitive treatment. ${ }^{1-5}$

The utility of MRCP to detect PD disruptions is widely

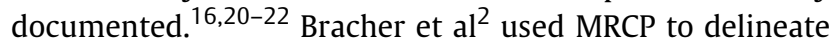
PD in patients with probable PD disruption and could accurately locate the site of disruption in 21 of 23 (91\%) patients. However, our study demonstrated that MRCP detected disruption in only about half of the patients. The most important limiting factor of MRCP in this setting is non-real-time visualization of PD filling and contrast extravasation as demonstrated on ERCP and also the non-availability of secretin in our country. ${ }^{20-23}$

The basis of conservative treatment is that decrement of pancreatic secretions will reduce the amount of pancreatic juice flowing through the disrupted PD and accelerate healing. ${ }^{24-26}$ Somatostatin analogs that reduce the pancreatic secretions and nasojejunal feeding are the main components of conservative therapy in patients with pancreatic ascites. ${ }^{25-27}$ Therapeutically large-volume paracentesis helps in reducing patient discomfort and might cause approximation of peritoneal surfaces of the lesser sac to the site of disruption, thereby sealing it. ${ }^{24} \mathrm{~A}$ study by Bakker et al ${ }^{9}$ showed success in fistula closure in three-fourths of patients in the conservative group and in more than $80 \%$ in the endotherapy group. Lower success rates (25-60\%) have been observed in studies that combined nil-per-oral, nasojejunal feeding, total parenteral nutrition, somatostatin analogs, and serial therapeutic paracentesis. ${ }^{25-28}$ In our study, endotherapy was successful in more than $80 \%$ of affected patients, out of which only $8 \%$ had a recurrence, one at 6 weeks and the other at 8 weeks after stent-removal. The success rate for conservative therapy was $60 \%$, out of which two-thirds had a recurrence on follow-up at 3 months. Pai et $\mathrm{al}^{29}$ reported complete resolution of ascites after endotherapy in $92.8 \%$ of patients over a median period of 5 weeks without any recurrence.

In our study, successful endotherapy had a significant association with the site of disruption in the body region and a bridging stent. This was similar to findings in a retrospective analysis by Varadrajulu et al. ${ }^{18}$ Additionally, concomitant pancreatic sphincterotomy was significantly associated with success in endotherapy in our study. Pai et $\mathrm{al}^{29}$ had a success rate of $96.4 \%$ on combining pancreatic sphincterotomy and stent placement during endotherapy. Pancreatic necrosis $>30 \%$ and the presence of intraductal calculi were significantly associated with a failed endotherapy. Jang et al. ${ }^{8}$ reported that necrosis $>50 \%$ was indirectly associated with a lower success rate of endotherapy thus requiring more surgical interventions. In the study by Varadrajulu et al. ${ }^{18}$ on 97 patients of PD disruption, a multivariate analysis showed that partially disrupted duct and stent bridging the disruption site were the factors crucial for the success of endotherapy. No statistically significant association between any of these factors and endotherapy outcome was seen on binary logistic regression analysis in our study. This might be because of the small sample size.

Reported adverse event rates in patients who underwent endoscopic therapy for PD disruption vary from $0-9 \%{ }^{18,30,31}$ It is still under evaluation whether early endotherapy is associated with detrimental effects in the future course of pancreatitis. ${ }^{18}$ Pai et al ${ }^{29}$ did endotherapy in 27 of 28 patients (96.4\%) at 3 weeks after symptom onset, but complications of pain, fever, and infection were seen in one-fourth of their patients. In our study, no major complication related to early endotherapy (within 2 weeks of symptom onset) was seen in the majority of the patients. Only one patient who had severe ANP and subsequent WON, did not improve even after two sessions of endoscopic necrosectomy, and died before any other intervention could take place. A recent study by Yokoi et $\mathrm{al}^{32}$ has shown that early pancreatic endotherapy is safe and also improves outcome. ${ }^{32}$ Discrepancies in reported adverse events can be explained by the lack of standard definitions of these conditions.

Surgery for pancreatic ascites is technically difficult because of the associated inflammation and concomitant PFCs. ${ }^{33,34}$ The major disadvantage is the high rate of complications and death ranging from 1 to $20 \%{ }^{34-36}$

Our study has several strengths. We showed that early endotherapy in patients with both ANP as well as CP is associated with a favorable outcome rather than continuing conservative therapy for a longer period and also that more extent of necrosis decreases the success rate of endotherapy. Similarly, this study showed that presence of intra-ductal calculi in CP is associated with failure in endotherapy for pancreatic ascites. Also, we demonstrated that a concomitant pancreatic sphincterotomy along with a stent bridging the disruption site especially in the body region increases the success rate of endotherapy. Based on the presence of rapidly refilling ascites and PD disruption on MRCP, we have proposed a simple algorithm to determine the timings of endotherapy with a high success rate in pancreatic ascites, as shown in -Fig. 1. Shortcomings of this study included it being a retrospective and uncontrolled analysis on a heterogeneous patient population.

To conclude, our study suggests that early endotherapy is effective and safe in the treatment of pancreatic ascites. Whether other modalities such as surgical, radiological, or any combination are more effective than endotherapy will only be answered by RCTs. But finding a large subset of comparable cases of this condition to conduct RCT is difficult due to its rarity.

NoteInstitutional review board approval number: IEC $34 / 20$.

Funding

None. 


\section{Conflict of Interest}

None declared.

\section{References}

1 Larsen M, Kozarek R. Management of pancreatic ductal leaks and fistulae. J Gastroenterol Hepatol 2014;29(07):1360-1370

2 Bracher GA, Manocha AP, DeBanto JR, et al. Endoscopic pancreatic duct stenting to treat pancreatic ascites. Gastrointest Endosc 1999;49(06):710-715

3 Sankaran S, Walt AJ. Pancreatic ascites: recognition and management. Arch Surg 1976;111(04):430-434

4 Cameron JL. Chronic pancreatic ascites and pancreatic pleural effusions. Gastroenterology 1978;74(01):134-140

5 Samanta J, Rana A, Dhaka N, et al. Ascites in acute pancreatitis: not a silent bystander. Pancreatology 2019;19(05):646-652

6 Baretti M, Pulluri B, Tsai HL, et al. The significance of ascites in patients with pancreatic ductal adenocarcinoma: a case-control study. Pancreas 2019;48(04):585-589

7 Rana SS, Sharma RK, Gupta R. Endoscopic management of pancreatic ascites due to duct disruption following acute necrotizing pancreatitis. JGH Open 2018;3(02):111-116

8 Jang JW, Kim MH, Oh D, et al. Factors and outcomes associated with pancreatic duct disruption in patients with acute necrotizing pancreatitis. Pancreatology 2016;16(06):958-965

9 Bakker OJ, van Baal MC, van Santvoort HC, et al; Dutch Pancreatitis Study Group. Endoscopic transpapillary stenting or conservative treatment for pancreatic fistulas in necrotizing pancreatitis: multicenter series and literature review. Ann Surg 2011;253 (05):961-967

10 van Dijk SM, Timmerhuis HC, Verdonk RC, et al; Dutch Pancreatitis Study Group. Treatment of disrupted and disconnected pancreatic duct in necrotizing pancreatitis: a systematic review and metaanalysis. Pancreatology 2019;19(07):905-915

11 Tanaka T, Kuroki T, Kitasato A, et al. Endoscopic transpapillary pancreatic stenting for internal pancreatic fistula with the disruption of the pancreatic ductal system. Pancreatology 2013;13 (06):621-624

12 Bhasin DK, Rana SS, Siyad I, et al. Endoscopic transpapillary nasopancreatic drainage alone to treat pancreatic ascites and pleural effusion.J Gastroenterol Hepatol 2006;21(06):1059-1064

13 Banks PA, Bollen TL, Dervenis C, et al; Acute Pancreatitis Classification Working Group. Classification of acute pancreatitis-2012: revision of the Atlanta classification and definitions by international consensus. Gut 2013;62(01):102-111

14 Bassi C, Dervenis C, Butturini G, et al; International Study Group on Pancreatic Fistula Definition. Postoperative pancreatic fistula: an international study group (ISGPF) definition. Surgery 2005; 138(01):8-13

15 Drake LM, Anis M, Lawrence C. Accuracy of magnetic resonance cholangiopancreatography in identifying pancreatic duct disruption. J Clin Gastroenterol 2012;46(08):696-699

16 Timmerhuis HC, van Dijk SM, Verdonk RC, et al. Various modalities accurate in diagnosing a disrupted or disconnected pancreatic duct in acute pancreatitis: a systematic review. Dig Dis Sci 2020; 66(05):1415-1424

17 Mortele KJ, Wiesner W, Intriere L, et al. A modified CT severity index for evaluating acute pancreatitis: improved correlation with patient outcome. Am J Roentgenol 2004;183(05):1261-1265

18 Varadarajulu S, Noone TC, Tutuian R, Hawes RH, Cotton PB. Predictors of outcome in pancreatic duct disruption managed by endoscopic transpapillary stent placement. Gastrointest Endosc 2005;61(04):568-575

19 Cotton PB, Lehman G, Vennes J, et al. Endoscopic sphincterotomy complications and their management: an attempt at consensus. Gastrointest Endosc 1991;37(03):383-393

20 O'Toole D, Vullierme MP, Ponsot P, et al. Diagnosis and management of pancreatic fistulae resulting in pancreatic ascites or pleural effusions in the era of helical CT and magnetic resonance imaging. Gastroenterol Clin Biol 2007;31(8-9 Pt 1):686-693

21 Soto JA, Alvarez O, Múnera F, Yepes NL, Sepúlveda ME, Pérez JM. Traumatic disruption of the pancreatic duct: diagnosis with MR pancreatography. Am J Roentgenol 2001;176(01):175-178

22 Panda A, Kumar A, Gamanagatti S, et al. Evaluation of diagnostic utility of multidetector computed tomography and magnetic resonance imaging in blunt pancreatic trauma: a prospective study. Acta Radiol 2015;56(04):387-396

23 Subramanian A, Dente CJ, Feliciano DV. The management of pancreatic trauma in the modern era. Surg Clin North Am 2007;87(06):1515-1532, $x$

24 Fernández-Cruz L, Margarona E, Llovera J, López-Boado MA, Saenz H. Pancreatic ascites. Hepatogastroenterology 1993;40(02): 150-154

25 Gislason H, Grønbech JE, Søreide O. Pancreatic ascites: treatment by continuous somatostatin infusion. Am J Gastroenterol 1991;86 (04):519-521

26 Parekh D, Segal I. Pancreatic ascites and effusion. Risk factors for failure of conservative therapy and the role of octreotide. Arch Surg 1992;127(06):707-712

27 Kurumboor P, Varma D, Rajan M, et al. Outcome of pancreatic ascites in patients with tropical calcific pancreatitis managed using a uniform treatment protocol. Indian J Gastroenterol 2009; 28(03):102-106

28 Chebli JMF, Gaburri PD, de Souza AF, et al. Internal pancreatic fistulas: proposal of a management algorithm based on a case series analysis. J Clin Gastroenterol 2004;38(09):795-800

29 Pai CG, Suvarna D, Bhat G. Endoscopic treatment as first-line therapy for pancreatic ascites and pleural effusion. J Gastroenterol Hepatol 2009;24(07):1198-1202

30 Gupta S, Gaikwad N, Samarth A, Sawalakhe N, Sankalecha T. Efficacy of pancreatic endotherapy in pancreatic ascites and pleural effusion. Med Sci (Basel) 2017;5(02):6

31 Neoptolemos JP, London NJ, Carr-Locke DL. Assessment of main pancreatic duct integrity by endoscopic retrograde pancreatography in patients with acute pancreatitis. Br J Surg 1993;80(01): 94-99

32 Yokoi Y, Kikuyama M, Kurokami T, Sato T. Early dual drainage combining transpapillary endotherapy and percutaneous catheter drainage in patients with pancreatic fistula associated with severe acute pancreatitis. Pancreatology 2016;16(04): 497-507

33 da Cunha JE, Machado M, Bacchella T, et al. Surgical treatment of pancreatic ascites and pancreatic pleural effusions. Hepatogastroenterology 1995;42(05):748-751

34 Selvakumar E, Vimalraj V, Rajendran S, et al. Pancreaticogastrostomy for pancreatic ascites. Hepatogastroenterology 2007;54 (75):657-660

35 Alexakis N, Sutton R, Neoptolemos JP. Surgical treatment of pancreatic fistula. Dig Surg 2004;21(04):262-274

36 Kaman L, Behera A, Singh R, Katariya RN. Internal pancreatic fistulas with pancreatic ascites and pancreatic pleural effusions: recognition and management. ANZ J Surg 2001;71(04):221-225 